\title{
A Technique to Measure the Spatial Quality of Slow Routes in Fragile Territories Using Image Segmentation
}

\author{
Andrea Rolando \\ Domenico D'Uva \\ Alessandro Scandiffio
}

Abstract

The current research aims at investigating the potential of image segmentation (IS) technology, based on web application, for measuring the spatial quality of slow routes. The big amount of street-level images, publicly available through several applications such as Mapillary, Google Street View, are relevant sources of information, that allow virtual explorations of many places around the world. The (IS) technology allows partitioning of a single digital image into sets of pixels in order to read and recognize the visual content within the frame of the image. By applying IS technology to the images taken along a defined route, it has established a method for grouping images in relation to their spatial features. The method has been applied to some stretches of slow-mobility routes, that are localized along the fragile coastal landscape of Trabucchi, south of Italy. A selection of images along the route, both in the outdoor and urban space, has been analyzed, with the aim to test the effectiveness of the method, able to produce useful information to define a Spatial Quality Index.

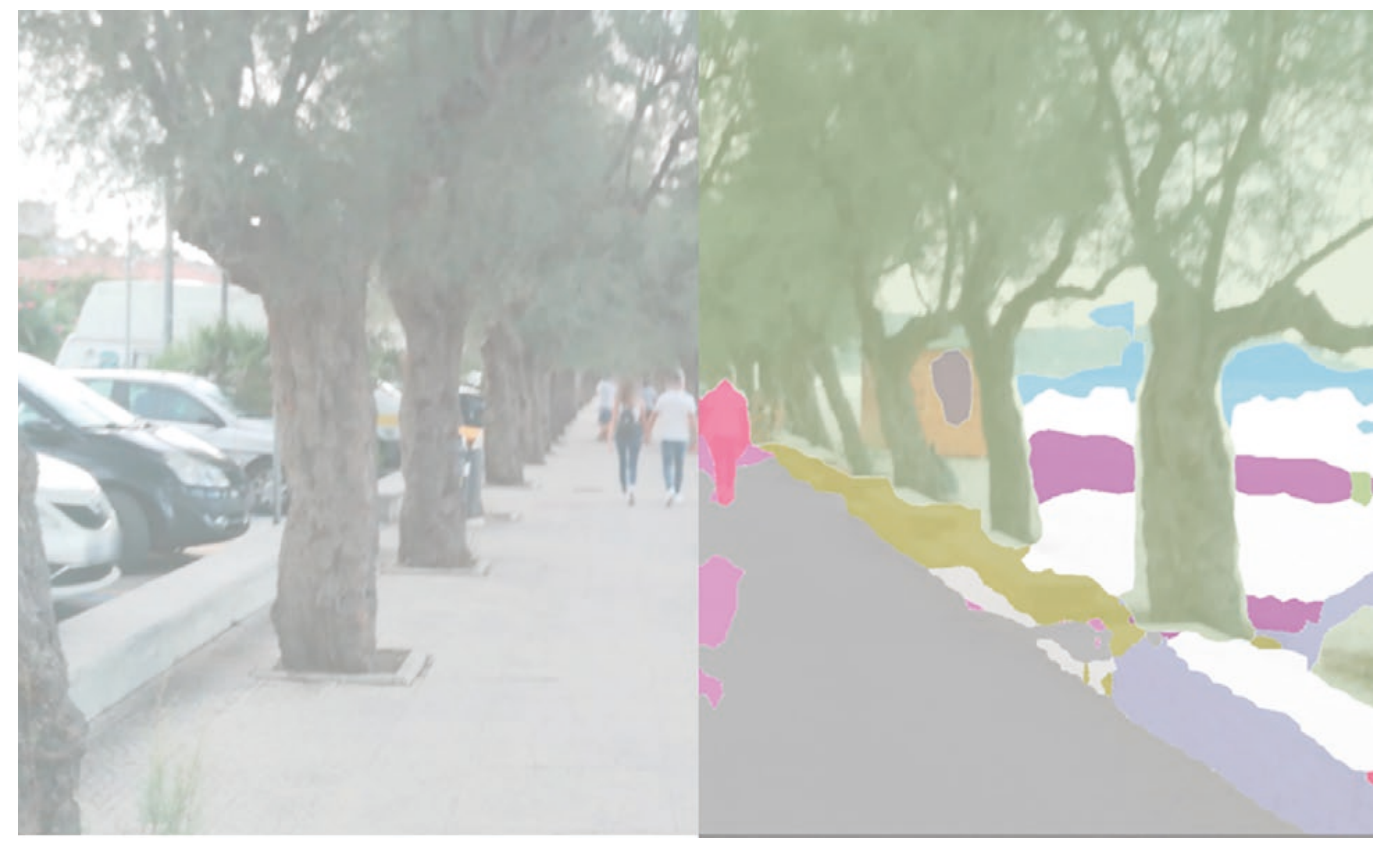




\section{Introduction}

The methodology presented in this paper results from a search for a solution to the problem of assessing the spatial quality of slow mobility routes [Scandiffio 2019; Bianchi et al. 2020], that are located off the beaten tracks, crossing territories that are marginal and distant from densely populated areas. The majority of studies on the subject refer to major urban centers, neglecting fragile areas of the territory. These areas are often characterized by a structural lack of data, which makes in-depth analysis difficult. However, it is possible to perform advanced research methods in the field of spatial analysis, by exploiting the potential of street-level imagery, users' generated contents that are available on the web portals. These methods belong to the Artificial Intelligence (Al) family. It is beyond the scope of this work to make a detailed analysis because, within this particular type of software, there are many subsets, each of which has a specific function [Zhang et al. 2018; Zhang et al. 2019; Cao et al. 2018]. The current research refers to a particular system of Machine Learning, named Deep Learning, that is based on Neural Networks [Buratti et al. 2020]. This particular subsystem allows analysing imagery and recognizing elements already present in an internal database of the machine. The use of an Al system is necessary because the database does not contain precisely the identified element, but only a series of similar elements that are analyzed and compared until an ideal candidate for the final recognition is found. The system, therefore, learns from the elements supplied to it and increases its knowledge step by step. Once the element has been recognized in the image, an even more specific procedure is used, called Image Segmentation (IS), which perimeters the object providing its position and area. Thus, this digital ecosystem returns the position and quantification of an element within a single image, which corresponds to a geo-referenced coordinate of the landscape. By performing the IS method to a sequence of geo-referenced imagery, it is possible to capture the landscape features along the route, which can be used for measuring its spatial quality. It is important to underline that the interpretation and assessment of the quality cannot be fully automated in the first instance: correct reading and interpretation of images must be prepared, defined and progressively tuned through an iterative process of machine training, possibly implemented and improved by massive users contributions. The process is in fact based on comparative assessments and still requires, in any case, a direct experience of places.

\section{Case Study}

The case study to which the methodology was applied is the Costa dei Trabucchi greenway in Abruzzo Region; a greenway converted into a tourist cycle route since 2017 [Marcarini, Rovelli 20 I8]. This route will stretch, upon completion, for 40 kilometers between Ortona and San Salvo. At the time of writing this work, it is almost wholly completed in two sections; the first to the north between Ortona and Fossacesia and the second to the south between Casalbordino and San Salvo. The northern section of this route was primarily constructed by the Adriatic railway line's retreat, leaving a void along the coast. It is currently almost completed and is accessible except for the tunnels, which are still under renovation. The southernmost section is a route that stitches together different territorial environments, the coastal towns of Casalbordino, Vasto, San Salvo, and the Regional Nature Reserve of Punta Aderci.This section has been entirely completed, except for the completely inaccessible tunnels. The coastline landscape along the route is very scenic, with wide and sandy beaches that alternate to natural cliffs that are interrupted by small urban settlements. Both sections are located protected natural areas of great value such as the Reserve of the Butterfly Cave and the Lecceta di Torino di Sangro and Trabucchi in San Vito, Fossacesia, and Vasto. For the purposes of the study, shots were selected in 6 sections belonging to the two sections. Often, the elements, that are in-between the cycle path and the sea, such as vegetation and houses, impede the landscape's unobstructed view, sometimes trapping the route in canyons in which the perception of the valuable elements of the landscape is seriously compromised. 


\section{Methodology}

The current methodology starts from the imagery surveying of the route, which has allowed to record the GPS track and to take photographs, orthogonal to the route direction, every 10 meters, by using the web application Mapillary [Porziy et al. 2020]. It is a web platform that automates mapping tools, by collecting street-level imagery taken by users with a standard smartphone. Mapillary exploits computer vision tools [Warburgy et al. 2020] and it allows the recognition system's application. The methodology is based on the object detection in images, based on IS technology. This technology is part of the digital ecosystem of Machine Learning that allows the recognition of the perimeter of objects and their measurements within the whole image. The objects are assimilated to the elements included in a training model already existing in the Machine Learning engine, and a percentage of occupation of the framed field of view is provided. An ad hoc algorithm has been written in Python language with the aim of extracting quantitative data related to each image. This algorithm makes it possible to shift the IS' heavy computational burden from local machines to remote cloud systems that operate the recognition in a much more efficient way. The image elements are extracted in the form of very rich JSON archives because they include the type of object, the area occupied in the image by each object, the recognition reliability percentage, and the coordinates of all the points that define the perimeter of the object. The algorithm skims all objects that occupy an area of less than $5 \%$ of the entire field of view, returning only the family and sub-family to which the object belongs, and the area occupied.

Fig. I. Workflow of the Image segmentation methodolgy for automatic recognition of landscape elements

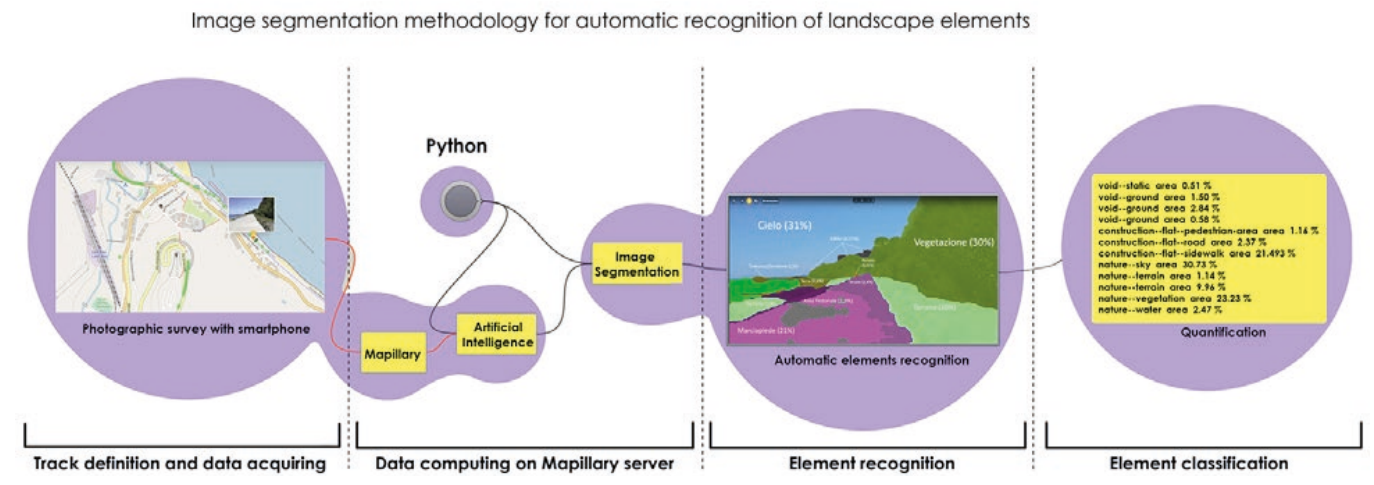

The grouping of images has been done by considering three main layouts as reference for the scenes. The layouts have been drawn, by selecting the horizontal surface of the path, the vertical obstructions and the openness of the sky. By assuming the horizontal surface of the path as an invariant component of all images, different thresholds of the area percentage of each component of the scene have been applied, in order to analyze the surfaces in the surrounding of the path, which, effectively, affect the landscape perception. Three different scene-types have been defined for grouping images. The scene-type I corresponds to an open environment, with small vertical elements on the sides and on the background of the image. The scene-type 2 corresponds to an environment where a wider surface of vertical elements is present only on one side. The scene-type 3 corresponds to a closer environment, surrounded by vertical elements on both sides. For each scene-type two different images, belonging to the outdoor and to the urban environment, have been selected, in order to test the effectiveness of the method. Both images of each scene-type have been selected in the same range of area percentage values (fig. 3). For each image it has been extracted the area percentage of each object in the image, through the Python algorithm. The raw values of area percentage, derived from the IS, have been grouped into eight main categories (path, sky, vegetation, edgings, water, terrain, buildings, mobile objects), in order to simplify the categorization and allow an immediate reading. For each category, it has been computed the area percentage. 


\section{Outcomes}

By processing the images with IS, three different thresholds have been identified for assessing the spatial quality of the landscape crossed by the route. The threshold values of the sky and vertical elements (vegetation, buildings, edgings) seem to be the most interesting indicators to outline the features of the scene.

The images in the scene-type I have the area percentage of the sky more than $40 \%$, and the sum of the area percentage of vertical surfaces (edgings, buildings, vegetation and mobile objects: people and cars) is less than $30 \%$. The images in the scene-type 2, the area percentage of the sky less than $40 \%$, and the sum of the area percentage of vertical surfaces is among $30 \%$ and $40 \%$. The images in scene-type n. 3 have the area percentage of the sky less than 20\%, while the sum of the area percentage of vertical surfaces is more than $40 \%$. The thresholds values identified in this research, could be modified and adapted, if they are tested in other environmental contexts.

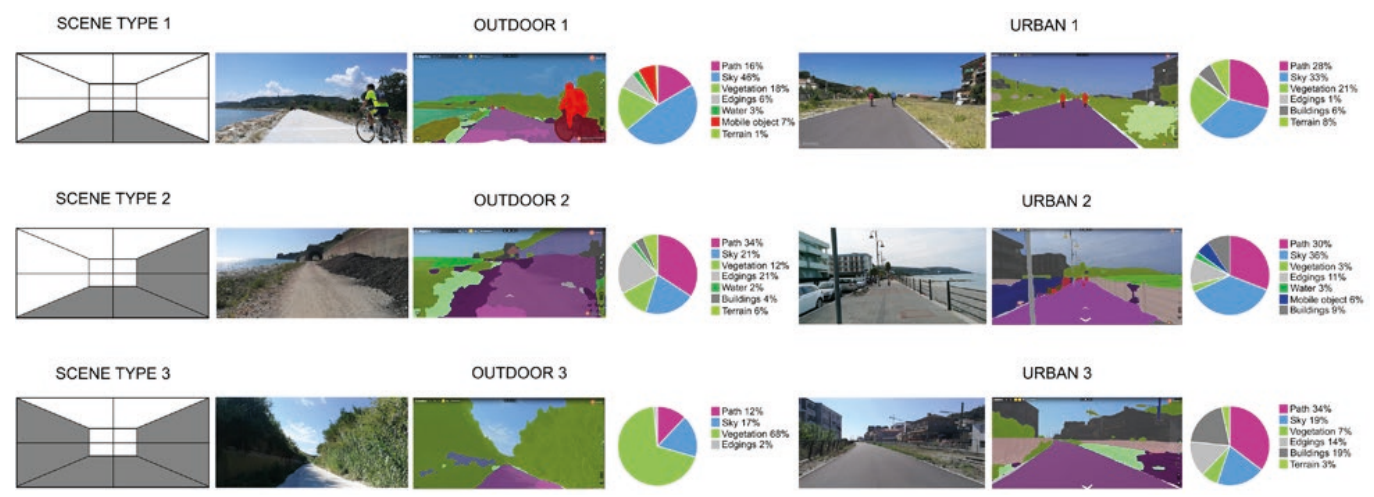

\section{Conclusion and Future Research Developments}

The limits of this methodology are twofold. The first is related to the system's problems, the second to the limitations of images data provision. The Mapillary system only allows the recognition of elements that can be read by referring to a database which is pre-defined by its developers and not open to changes. Therefore, some elements typical of the area covered by the case study are not taken into account. For example, the trabucchi, amphibious machines that are the main heritage - from which the coast itself takes its name - are selected as Void objects, not recognized. These objects along with other notable features, are not recognized because the Mapillary database only includes standard features. It would be necessary to customize the database to select only those objects that are expected to be retrieved according to their relevance for our specific qualitative analysis. Such an update of the tool could take advantage of the peculiar characteristic of Machine Learning systems, namely the ability to learn through analysis, and improve its precision and reliability. However, the problem arises when training this new database, whose operation requires significant machine time resources and the need to rely on external structures to complete the process. During the analysis of the case studio, problems were encountered in the recognition system because only 100 items per image are analyzed at any one time. The main features could be included in this value, but some attempts at element recognition only found $51 \%$ of them. This limitation can be overcome by directing the system to search for single categories of objects, instead of the totality, by expanding the number of detectable elements. To overcome this first type of limitations, the evolution of methodology will consist of updating the Python algorithm of parameter extraction, e.g., implementing the grouping of the elements recognized in the indicated categories. This procedure would start from the sum of the elements with equal features to automatically group and draw a graph of what is currently done manually.

The second type of limitation concerns how photographs are taken. A positive aspect of Mapillary is the possibility to survey using a standard smartphone. This flexibility implies a significant problem for recognition: namely the angle between the direction (perpendicular, along the lens axis to the smartphone sensor) and the horizon. Tilting the field of view downwards increases the amount of land (increasing the number of elements included and incurring in the problem told above) in 
the frame.Tilting it in the opposite direction increases the percentage of the sky that fills the frame. The incidence of this factor will be the subject of future research developments. A further limitation of this type is the standardization of the photos because they are taken with various instruments with different resolutions and fields of view, with intuitively different results. The differences induced by these limitations can be overcome by using standardized tools for capturing photos, implementing a camera whose inclination, position, and shock-absorber can be controlled within specific well-defined parameters. A completely different approach to this issue is relying on more reliable photo databases such as Google Street View [Zhanga et al. 2020; Anguelov et al. 2020] but with limited coverage of the slow mobility routes covered in this work. A better definition of the thresholds for each category can be found, in order to apply the method to other territorial contexts. Related to this problem is the limited portion of the space that is framed by the images. In fact, this method only allows to detect the landscape elements that are framed in the images. The application of other methods that define landscape components extracting them from GIS database could be integrated in the procedure. The best foreseen solution could aim to define the most relevant components of a landscape extracting and defining them both from GIS databases (made of geometric entities derived from visual knowledge) and from Image Segmentation procedures, that feed a similar database, but using visual recognition of the same entities. In the end this double checked definition of entities should assure the correct definition of each item. Further developments could investigate the possibility to define a set of relevant objects referred to geometric entities (points, lines, surfaces) to be considered as common database records, so to be recognized and confronted both in the image segmentation based process and in its complementar GIS based method of analysis.

\section{Acknowledgments}

This work has been carried on within the activities of the E-scapes research group (www.e-scapes.polimi.it directed by A. Rolando) and the Territorial Fragilities Program - MapFrag group (www.eccellenza.dastu.polimi.it) of the Department of Architecture and Urban Studies.

\section{References}

Anguelov Dragomir, Dulong Carole, Filip Daniel, Frueh Christian, Lafon Stéphane, Lyon Richard, Ogale Abhijit S. ,Vincent Luc, Weaver Josh (20 10). Google Street View: Capturing the World at Street Level. In Computer, 43(6,) pp. 32-38.

Bianchi Alessandro, D'Uva Domenico, Rolando Andrea, Scandiffio Alessandro (2020). A View from the Track: Measuring Spatial Quality of Slow Mobility Routes. Possible Integration of GIS and Machine Learning Based Methods. In Connettere Connecting. Un disegno per annodare e tessere/drawing for weaving relationships. Milano: FrancoAngeli, pp. 2973-2980.

Cao Rui, Zhu Jiasong,Tu Wei, Li Qingquan (20 I 8). Integrating Aerial and StreetView Images for Urban Land Use Classification. In Remote Sensing, 10, pp. I-23.

Marcarini Albano, Rovelli Roberto (2018). Atlante Italiano delle ferrovie in disuso. Firenze: Istituto Geografico Mllitare.

Porziy Lorenzo, Hofingerz Markus, Ruiz Idoia, Serrat Joan, Rota Bul’oy Samuel, Kontschiedery Peter (2020). Learning MultiObject Tracking and Segmentation from Automatic Annotations. In Computer Vision and Pattern Recognition, arXiv.

Scandiffio Alessandro (2019). Mapping spatial quality of slow routes with a GIS-based method. A comparative assessment of alternative routes. In International Archives Photogrammetry Remote Sensing Spatial Information Science, XLII-2 W I 5, pp. I 07 I - I 076.

Warburgy Frederik, Haubergy Søren, Lopez-Antequeraz Manuel, Gargalloz Pau, Kuangz Yubin, Civerax Javier (2020). Mapillary Street-Level Sequences: A Dataset for Lifelong Place Recognition. In Conf. on Computer Vision and Pattern Recognition (CVPR), pp. I- I0.

Zhang Fan, Zhou Bolei, Ratti Carlo (2019). Discovering place-informative scenes and objects using social media photos. In Royal Society open science, 6 (3).

Zhang Fan, Zhou Bolei, Liu Liu, Liu Yu, Fung Helene H. , Lin Hui, Ratti Carlo (20 I 8). Measuring human perceptions of a largescale urban region using machine learning. In Landscape and Urban Planning, I 80, pp. I48- I60.

Zhang Fan, Zuc Jinyan, Huc Mingyuan, Zhub Di, Kangd,Yuhao, Gaod Song, Zhang Yi, Huang Zhou (2020). I0 478 Uncovering inconspicuous places using social media check-ins and street view images. In Computer, Environment and Urban Systems. Amsterdam: Elsevier.

\section{Authors}

Andrea Rolando, Dept.of Architecture and Urban Studies, Politecnico di Milano, andrea.rolando@polimi.it Domenico D'Uva, Dept of Architecture and Urban Studies, Politecnico di Milano, domenico.duva@polimi.it Alessandro Scandiffio, Dept. of Architecture and Urban Studies, Politecnico di Milano, alessandro.scandiffio@polimi.it 
